\title{
An Impossibility Theorem for Allocation Aggregation
}

\section{Carl Wagner and Mark Shattuck (in press, Journal of Philosophical Logic, 2014)}

\begin{abstract}
Among the many sorts of problems encountered in decision theory, allocation problems occupy a central position. Such problems call for the assignment of a nonnegative real number to each member of a finite (more generally, countable) set of entities, in such a way that the values so assigned sum to some fixed positive real number $s$. Familiar cases include the problem of specifying a probability mass function on a countable set of possible states of the world $(s=1)$, and the distribution of a certain sum of money, or other resource, among various enterprises. In determining an $s$-allocation it is common to solicit the opinions of more than one individual, which leads immediately to the question of how to aggregate their typically differing allocations into a single "consensual" allocation. Guided by the traditions of social choice theory (in which the aggregation of preferential orderings, or of utilities is at issue) decision theorists have taken an axiomatic approach to determining acceptable methods of allocation aggregation. In such approaches so-called "independence" conditions have been ubiquitous. Such conditions dictate that the consensual allocation assigned to each entity should depend only on the allocations assigned by individuals to that entity, taking no account of the allocations that they assign to any other entities. While there are reasons beyond mere simplicity for subjecting allocation aggregation to independence, this radically anti-holistic stricture has frequently proved to severely limit the set of acceptable aggregation methods. As we show in what follows, the limitations are particularly acute in the case of three or more entities which must be assigned nonnegative values summing to some fixed positive number $s$. For if the set $V \subseteq[0, s]$ of values that may be assigned to these entities satisfies some simple closure conditions and (as is always the case in practice) $V$ is finite, then independence allows only for dictatorial or imposed (i.e., constant) aggregation. This theorem builds on and extends a theorem of Bradley and Wagner [6] and, when $V=\{0,1\}$, yields as a corollary an impossibility theorem of Dietrich [8] on judgment aggregation.
\end{abstract}

Keywords Allocation Aggregation · Dictatorship · Judgment Aggregation · Probability

1. Allocation Aggregation and Independence. Among the many sorts of problems encountered in decision theory, allocation problems occupy a central position. Such problems call for the assignment of a nonnegative real number to each member of a finite (or more generally, countable) set of entities, in such a way that the values so assigned sum to some fixed positive real number $s$. When the entities in question constitute a set of pairwise mutually exclusive, exhaustive states of the world, and $s=1$, such an allocation is called a probability mass 
function. ${ }^{1}$ When $s$ designates a certain sum of money, or other material resource, and the entities in question are various enterprises, such an allocation distributes (all of) the resource in question among those enterprises. The terms of an allocation problem may dictate certain restrictions on the allocated amounts, or allow them to be totally unrestricted, except for the constraint on their sum. $^{2}$

In determining an $s$ - allocation, it is common to solicit the opinions of more than one individual, which leads immediately to the question of how to aggregate their typically differing allocations into a single "consensual" allocation. In this matter, decision theorists have followed the lead of social welfare theorists such as Kenneth Arrow [3] in approaching the problem axiomatically. In axiomatic approaches to allocation aggregation, so-called independence ${ }^{3}$ conditions have been ubiquitous. Such conditions dictate that the "consensual" allocation value assigned to a given entity should depend only on the values assigned by individuals to that entity, taking no account of the values that they assign to any other entities. It should be noted, however, that the adoption of such a radically anti-holistic stricture on the synthesis of expert opinion has been motivated by reasons beyond mere simplicity. McConway [13] has shown, for example, that an aggregation method for probability measures commutes with marginalization to sub-sigma algebras precisely when the "consensual" probability assigned to each event $E$ depends only on $E$ and the probabilities assigned to $E$ by the experts in question. In the case of judgment aggregation, where individuals are limited to assigning propositions one of the values true or false, Dietrich [8] has shown that independence conditions of varying strength correlate with immunity of an aggregation method to various types of agenda manipulation. On a different note, Wagner ([17], [18]) has argued that independence conditions (or variations thereof allowing for normalization after entity-by-entity aggregation) are a predictable concomitant of universal domain conditions, which require of an aggregation method that it be applicable to every logically possible "profile" of individual opinions.

On the other hand, subjecting allocation aggregation to an independence requirement has frequently proved to severely limit the set of acceptable aggregation methods. In what follows we demonstrate that independence places particularly acute restrictions on the aggregation of $s$-allocations to three or more entities when the valuation domain (viz., the subset of values in $[0, s]$ that may be assigned to these entities) is finite and satisfies some simple closure conditions. For subjecting the aggregation of such $s$-allocations to independence (under the assumption of universal domain) allows only for dictatorial or imposed aggregation. Our results build on and extend work of Bradley and Wagner [6] and yield as a corollary an impossibility theorem for judgment aggregation due to Dietrich [8].

2. The Valuation Domain of an Allocation Problem. In what follows, we denote by $V$ the aforementioned valuation domain associated with a given allocation problem. We shall assume that any such domain satisfies the following minimal closure conditions: 


$$
\begin{aligned}
& 0 \in V, \\
& x \in V \Rightarrow s-x \in V, \text { and } \\
& x, y \in V \text { and } x+y \leq s \Rightarrow x+y \in V .
\end{aligned}
$$

Remarkably, the subsets $V$ of $[0, s]$ satisfying the above conditions fall into just two radically distinct categories. A set $V$ is dense in $[0, s]$ if, for all $x, y \in[0, s]$ such that $x<y$, there exists a $v \in V$ such that $x<v<y$.

THEOREM 2.1. A subset $V$ of $[0, s]$ satisfying (2.1), (2.2), and (2.3) is either dense in $[0, s]$, or finite.

Proof. See Appendix A.

Remark 2.1.1. A set $E$ of real numbers is discrete if, for every $x \in E$, there exists an $\varepsilon>0$ such that $\{y:|y-x|<\varepsilon\} \cap E=\{x\}$. Clearly, no discrete subset of $[0, s]$ can be dense in $[0, s]$. So Theorem 2.1 has as a corollary the fact that any discrete valuation domain satisfying (2.1)-(2.3) must be finite.

3. Allocation Aggregation Theorems. We now consider the problem of aggregating the $s$-allocations of individuals $i=1, \ldots, n$ to entities $j=1, \ldots, m .{ }^{4}$ Let us record the opinions of the these individuals in an $n \times m$ matrix $A=\left(a_{i, j}\right)$, where $a_{i, j}$ records the value assigned by individual $i$ to entity $j$. If the values $a_{i, j}$ are restricted to belonging to the set $V$, we call such a matrix an $s$-allocation matrix in $V$, and we denote the set of all such matrices by $A(n, m ; V)$. We call a vector $\left(a_{1}, \ldots, a_{m}\right)$ with each $a_{j} \in V$ and $a_{1}+\cdots+a_{m}=s$ an $s$-allocation vector in $V$, and we denote the set of all such vectors by $A(m ; V)$. In what follows, the $j^{\text {th }}$ column of a matrix $A$ is denoted by $A_{j}$, and the $j^{\text {th }}$ entry of the row vector $a$ is denoted by $a_{j}$. The $n \times 1$ column vector with all entries equal to $c$ will be designated by the boldface symbol $\mathbf{c}$. An allocation aggregation method (AAM) is any function $F: A(n, m ; V) \rightarrow A(m ; V)$. Each AAM $F$ furnishes a method of reconciling the possibly different opinions recorded in an $s$-allocation matrix $A$ in the form of the group assignment $F(A)=a=\left(a_{1}, \ldots, a_{m}\right)$. As noted earlier, aggregation theory has followed social choice theory in adopting an axiomatic approach to the study of AAMs. Here we wish to focus on the following axiomatic restriction on aggregation:

Independence (I). For all $j \in\{1, \ldots, m\}$, and all $A, B \in A(n, m ; V), A_{j}=B_{j} \Rightarrow F(A)_{j}=F(B)_{j}$. 
Remark. In specifying that the domain of any AAM must be equal to the set $A(n, m ; V)$ of all $s$ - allocation matrices, we are implicitly postulating what is often called the Universal (or Unrestricted) Domain Condition. In specifying that the co-domain of any AAM must be equal to the set $A(m ; V)$ of all $s$-allocation row vectors, we are implicitly postulating what is often called the Collective Rationality Condition. Alternatively, we might have defined an AAM to be a function $F: D \rightarrow \mathbb{R}^{\mathrm{m}}$, where $D$ is a non-empty subset of $A(n, m ; V)$, and postulated Universal Domain and Collective Rationality as explicit axioms.

Under the assumption that the domain of any AAM is equal to $A(n, m ; V)$, condition I is clearly equivalent to the existence of functions $f_{j}: V^{n} \rightarrow V, j=1, \ldots, m$, such that, for all $A \in A(n, m ; V), F(A)_{j}=f_{j}\left(A_{j}\right)$ and $\sum_{j=1}^{m} f_{j}\left(A_{j}\right)=s .{ }^{5}$ This condition thus dictates that, for each $j \in\{1, \ldots, m\}$, the value assigned to entity $j$ by the group, should depend (via $f_{j}$ ) only on the values $a_{1, j}, a_{2, j}, \ldots, a_{n, j}$ assigned by individuals to that variable, independently of the values that they assign to other variables.

The following theorem characterizes those AAMs satisfying condition I when $V$ comprises the entire interval $[0, s]$.

THEOREM 3.1. If $n \geq 2$ and $m \geq 3$, an AAM $F: A(n, m ;[0, s]) \rightarrow A(m:[0, s])$ satisfies the independence condition $\mathrm{I}$ if and only if there exist "weights" $w_{1}, \ldots, w_{n}$ in the interval $[-1,1]$ and real numbers $s_{1}, \ldots, s_{m}$ in $[0, s]$ satisfying

$$
-s \sum^{-} w_{i} \leq s_{j} \leq s\left(1-\sum^{+} w_{i}\right) \quad j=1, \ldots, n
$$

where $\sum^{-}$indicates the sum of the negative weights and $\sum^{+}$the sum of the positive weights, and

$$
\sum_{j=1}^{m} s_{j}=\left(1-\sum_{i=1}^{n} w_{i}\right) s
$$

such that, for all $A=\left(a_{i, j}\right) \in A(n, m ;[0, s])$,

$$
F(A)_{j}=f_{j}\left(A_{j}\right)=\sum_{i=1}^{n} w_{i} a_{i, j}+s_{j}
$$

Proof. See Aczél, et al [1]. 
Remark 3.1.1. It is noteworthy that the weights here may take negative values, subject to conditions (3.1) and (3.2). In particular, (3.1) implies that $\sum\left|w_{i}\right| \leq 1$, and hence that $\sum w_{i} \leq 1$. Note also that the weights remain invariant across different values of $j$. If $\sum w_{i}=1$, then $s_{j}=0$ for all $j$, and so $w_{i} \geq 0$ for all $i{ }^{6}$ So aggregation here differs from simple weighted arithmetic averaging if and only if $\sum w_{i}<1$. In such cases, (3.3) may be recast in the form

$$
\begin{aligned}
& F(A)_{j}=f_{j}\left(A_{j}\right)=\sum_{i=1}^{n} w_{i}\left(a_{i, j}-\sigma_{j}\right)+\sigma_{j}=\sum_{i=1}^{n} w_{i} a_{i, j}+\left[1-\sum_{i=1}^{n} w_{i}\right] \sigma_{j}, \text { where } \\
& \sigma_{j}=s_{j} /\left(1-\sum_{i=1}^{n} w_{i}\right) \geq 0 \text { and } \sum_{j=1}^{m} \sigma_{j}=s .
\end{aligned}
$$

Aggregation carried out by (3.4) might arise when experts report to an external decision maker who incorporates his or her own preferred allocation $\left(\sigma_{1}, \ldots, \sigma_{m}\right)$ into the final allocation.

Remark 3.1.2. The prototype of Theorem 3.1 (and all related aggregation theorems involving weighted arithmetic averaging) is the characterization of weighted arithmetic means in Aczél and Wagner [2], which involves, unsurprisingly, solving the Cauchy functional equation $f(x+y)=f(x)+f(y)$.

Remark 3.1.3. When $s=1$ and the entities under consideration constitute a set of possible states of the world, Theorem 3.1 characterizes the methods for aggregating the probability mass functions (see note 1, supra) of several individuals subject to condition I. Genest [10] has proved analogous results for the aggregation of probability measures defined on a fixed sigma algebra. There is a large literature on the aggregation of probabilistic judgments. Genest and Zidek [11] furnish a useful annotated bibliography of work done through the mid-1980s. Of particular note is the beautiful paper of McConway [13], mentioned briefly in Section 1. ${ }^{7}$

Remark 3.1.4. It is natural to wonder if Theorem 3.1 holds for any other dense subsets of [0, $s]$. When $s$ is rational, $\mathrm{V}$ consists of all rational numbers in $[0, s]$, and the weights $w_{i}$ and quantities $s_{j}$ are rational, then formula (3.3) is clearly sufficient for independence. The same is true, mutatis mutandis, for certain other families of rational numbers in $[0, s]$, given that they satisfy the additional closure property (iv) $x, y \in V$ and $x y \leq s \Rightarrow x y \in V$. We have been unable, however, to determine in any of these cases whether aggregation by a formula like (3.3) is necessary for independence.

Remark 3.1.5. Although aggregation methods furnished by (3.3) and (3.4) accommodate condition I, they typically fail to commute with Jeffrey conditioning, or to preserve agreed-upon cases of stochastic independence (Wagner, [16], [17]). So, while admitting a non-trivial class of 
aggregation methods for certain valuation domains that are dense in $[0, s]$, condition I still poses some unfortunate restrictions on such methods.

Suppose now that $V$ fails to be dense in $[0, s]$ and is consequently (by Theorem 2.1) finite. We say that an AAM $F$ is dictatorial if there exists an individual $d \in\{1, \ldots, n\}$ such that, for all $A \in A(n, m ; V), F(A)=\left(a_{d, 1}, \ldots, a_{d, m}\right) ; F$ is imposed (or constant) if there exists an $s$-allocation row vector $a=\left(a_{1}, \ldots, a_{m}\right)$ such that, for all $A \in A(n, m ; V), F(A)=a$. Assuming that both dictatorial and imposed aggregation are unacceptable, the following constitutes an impossibility theorem for allocation aggregation:

THEOREM 3.2. Suppose that the valuation domain $V \subseteq[0, s]$ is finite, and satisfies the closure conditions (2.1)-(2.3). If $n \geq 2$ and $m \geq 3$, an AAM $F: A(n, m ; V) \rightarrow A(m ; V)$ satisfies the independence condition $I$ if and only if $F$ is dictatorial or imposed.

Proof. See Appendix B.

Remark 3.2.1. When $m=2$, every AAM trivially satisfies condition I.

Remark 3.2.2. If, in addition to condition I, one postulates Zero Unanimity Preservation (for all $j \in\{1, \ldots, m\}$ and all $\left.A \in A(n, m ; V), A_{j}=\mathbf{0} \Rightarrow F(A)_{j}=0\right)$, then only dictatorial aggregation is allowed, as shown in Bradley and Wagner [6].

Given a valuation domain $V \subseteq[0, s]$, a number $x \in[0, s]$ is isolated from $V$ if there exists an $\varepsilon>0$ such that $(x-\varepsilon, x+\varepsilon) \cap V=\varnothing$. The following theorem unifies the results of Theorems 3.1 and 3.2:

THEOREM 3.3. Suppose that the valuation domain $V \subseteq[0, s]$ satisfies the closure conditions (2.1)-(2.3), and that $n \geq 2$ and $m \geq 3$. If either (i) $V=[0, s]$ or (ii) there exists an $x \in[0, s]$ which is isolated from $V$, then an AAM $F: A(n, m ; V) \rightarrow A(m ; V)$ satisfies the independence condition I if and only if $F$ is affine, i.e., given by a formula of the form (3.3).

Proof. Sufficiency: obvious. Necessity: When $V=[0, s]$, this is Theorem 3.1. When there exists an $x \in[0, s]$ which is isolated from $V$, then $V$ fails to be dense in $[0, s]$. Hence $V$ is finite by Theorem 2.1, and $F$ is dictatorial or imposed by Theorem 3.2. Formula (3.3) represents a dictatorship when $s_{j}=0$ for all $j$, and there exists $d \in\{1, \ldots, n\}$ such that $w_{d}=1$ and $w_{i}=0$ for all $i \neq d$. Formula (3.3) represents a case of imposed aggregation when $w_{i}=0$ for all $i$, and $s_{1}, \ldots, s_{m}$ are numbers in $V$ summing to $s$. 
Remark 3.3.1. The prototype of Theorem 3.3 is Theorem 1 of Dietrich and List [9], in which it is demonstrated that, under certain axioms, including, in particular, independence, an aggregation rule for binary or cardinal judgments must be linear.

\section{An Application to Judgment Aggregation}

Following Dietrich [8], call a set of well-formed formulas (wffs) $X$ of the propositional calculus an agenda if $X$ is closed under logical negation. ${ }^{8}$ Denote by $\mathbf{J}$ the set of all subsets ("judgment sets") $J$ of $X$ that are consistent (there is an assignment of truth values to the propositional variables occurring in the wffs of $J$ for which every wff in $J$ is true) and complete (for each wff $p$ in $X$, either $p \in J$ or $\neg p \in J)$. A function $\Phi: \mathbf{J}^{\mathrm{n}} \rightarrow \mathbf{J}$ is called a judgment aggregation rule, and furnishes a method of combining the complete, consistent judgment sets $J_{1}, \ldots, J_{n}$ of $n$ individuals into a single complete, consistent judgment set $J=\Phi\left(J_{1}, \ldots, J_{n}\right)$. In selecting $J_{i}$ as his or her judgment set, individual $i$ assigns each wff in $J_{i}$ the truth value 1 (designating "true") and each wff in the agenda which is not in $J_{i}$ the truth value 0 (designating "false"). Associated with each "profile" of judgment sets $\left(J_{1}, \ldots, J_{n}\right)$ and each wff $p \in X$ is an $n$-tuple $\left(t_{J_{1}, p}, \ldots, t_{J_{n}, p}\right) \in\{0,1\}^{n}$, where $t_{J_{i}, p}=1$ if and only if $p \in J_{i}$. The judgment aggregation rule $\Phi$ is said to arise from proposition-wise aggregation if, for each $p \in X$, there exists a mapping $M_{p}:\{0,1\}^{n} \rightarrow\{0,1\}$ such that for all profiles $\left(J_{1}, \ldots, J_{n}\right) \in \mathbf{J}^{\mathrm{n}}, p \in \Phi\left(J_{1}, \ldots, J_{n}\right)$ if and only if $M_{p}\left(t_{J_{1}, p}, \ldots, t_{J_{n}, p}\right)=1$. A wff $\alpha \in X$ is an atom of $X$ if $\alpha$ is not contradictory, and for every

$q \in X, \alpha \vDash \mathrm{q}$ or $\alpha \vDash \neg q$. The agenda $X$ is atomic if each wff in $X$ that is not contradictory is logically equivalent to a disjunction of atoms of $X$.

The following result (Dietrich [8, Corollary 2]) follows from Theorem 3.2. (wffs $p$ and $q$ are said to be in trivial dependence if $p$ is logically equivalent to $q$ or to $\neg q$, or if $p$ or $q$ is a tautology or a contradiction.)

THEOREM 4.1. If $X$ is atomic and contains at least two wffs not in trivial dependence, then a non-constant judgment aggregation rule $\Phi$ arises from proposition-wise aggregation if and only if $\Phi$ is dictatorial (i.e., $\exists d \in\{1, \ldots, n\}$ such that, for all $\left.\left(J_{1}, \ldots, J_{n}\right) \in \mathrm{J}^{\mathrm{n}}, \Phi\left(J_{1}, \ldots, J_{n}\right)=J_{d}\right)$.

Proof. Sufficiency: Obvious. Necessity: Suppose first that $X$ is finite. If $p$ and $q$ are wffs of $X$ not in trivial dependence, then, for example, $p$ and $\neg p$ cannot both be atoms of $X .{ }^{9}$ It then follows from atomicity of $X$ that there are at least three atoms in $X$. Each complete, consistent judgment set $J$ contains precisely one of these atoms, namely, $\alpha=\wedge_{p \in J} p$, whence $J=\{p \in X: \alpha \neq p\}$. Hence, there is an obvious one-to-one correspondence between $\mathbf{J}^{\mathrm{n}}$ and 
$A(n, m ;\{0,1\})$, as well as between the set of judgment aggregation rules $\Phi: \mathbf{J}^{\mathrm{n}} \rightarrow \mathbf{J}$ arising from proposition-wise aggregation and the set of allocation aggregation methods $F: A(n, m ;\{0,1\}) \rightarrow A(m ;\{0,1\})$ satisfying the independence condition $\mathrm{I}$, with dictatorial judgment aggregation rules corresponding to dictatorial allocation aggregation methods. The asserted result now follows from Theorem 3.2.

If $X$ is infinite, let $A \subseteq X$ be the set of atoms in $X$. As noted above, there is at least one finite set $B \subseteq A$ containing at least three atoms. For any such set $B$, consider the sub-agenda $\{p, \neg p: p \in B\}$. The aggregation rule $\Phi$ induces an aggregation rule for each such sub-agenda, with the induced rule inheriting the property of proposition-wise aggregation from $\Phi$. By the argument in the preceding paragraph, each of these induced rules is either dictatorial or constant. It is easily verified that either all of these rules are constant, or all of these rules are dictatorial, with the same dictator in each case. Hence the original rule for $X$ is either constant or dictatorial.

Remark 4.1.1. . Note that the case $V=\{0,1\}$ of allocation aggregation does not directly yield a case of judgment aggregation, since the set of states of the world does not constitute an agenda, failing, as it does, to be closed under negation. So Theorem 4.1, while a corollary of Theorem 3.2 , is not simply a special case of Theorem 3.2, both for the aforementioned reason, and because Theorem 4.1 holds for infinite, as well as finite, agendas.

5. Discussion. Of course, impossibility theorems (or other theorems that narrowly circumscribe the set of acceptable aggregation methods) may no longer hold sway under weaker axioms of aggregation. Two obvious ways of weakening such axioms are to $(i)$ narrow the domain or (ii) enlarge the co-domain of the aggregation functions under consideration. In social welfare theory, for example, Black [5] has proved that Arrow's impossibility theorem no longer holds if voters' preferential orderings are "single peaked," and Brown [7] has shown that Arrow's axioms can be satisfied if the societal ordering produced by a social welfare function need only be acyclic. In the realm of probability aggregation, the limitation to aggregation by weighted arithmetic means (as in [12] and [13]) can be evaded, while still satisfying state-wise aggregation , if one is willing to countenance certain lower or upper probabilities in the co-domain. ${ }^{10}$

However, the aforementioned strategies do not appear to offer much in the way of consolation with respect to Theorem 3.2. If $V=\left\{\frac{i s}{r}: i=0, \ldots, r\right\}$ and $V^{+}=\left\{\frac{i s}{k r}: i=0, \ldots, k r\right\}$, where $k$ is a positive integer that is strictly greater than 2 , then of course there are non-dictatorial, nonimposed AAMs $F: A(n, m ; V) \rightarrow A\left(m ; V^{+}\right)$satisfying condition I. ${ }^{11}$ This gambit may even be reasonable when the goal is to allocate a fixed sum of money among competing projects. But it appears especially inapt in the case of aggregating probability mass functions, and even more so in the case of judgment aggregation. ${ }^{12}$ Another possible way of dodging Theorem 3.2 might be to aggregate the values assigned to each entity using weighted arithmetic averaging, and then 
round the resulting assignments so that they are elements of $V$, while still summing to $s$. But this would require one to select and defend a rounding policy, the difficulty of which has been amply illustrated in the problem of allocating seats in a legislature (see, e.g., [4]).

It should be noted that the foregoing strategies all persist in satisfying some sort of independence condition. Would dropping independence open up the canon of acceptable aggregation methods? It is difficult to see how to avail oneself of the freedom from independence if one insists on maintaining the requirement of universal domain. ${ }^{13}$ If one gives up both independence and universal domain, however, the outlook is, at least in certain cases, more promising. It is shown in [17], for example, that (stochastic) independence-preserving probability aggregation methods can be constructed if such methods need only apply to probability profiles exhibiting a particular type of independence, and one dispenses with the requirement of state-wise aggregation. In a particularly interesting example from the theory of judgment aggregation, Dietrich [8] has exhibited an aggregation method that satisfies independence only for certain sets of logically independent "premises" and generates consistent collective judgment sets.

As things presently stand, however, these strategies do not seem to offer much help in dodging Theorem 3.2, for there appear to be no natural proper subsets of $A(n, m ; V)$ on which one might exploit the freedom from entity-wise aggregation to devise an aggregation method with codomain $A(m ; V)$. While this state of affairs persists, Theorem 3.2 would appear to have genuine limitative implications for the goal of devising a rational method of allocation aggregation for finite valuation domains.

\section{APPENDIX A}

In preparation for what follows, let us recall a few basic topological concepts. If $x$ is any real number and $\varepsilon>0$, the open neighborhood $N_{\varepsilon}(x)=\{y:|y-x|<\varepsilon\}$. If $E$ is any set of real numbers, a real number $x$ (which may or may not be an element of $E$ ) is an accumulation point of $E$ if, for every $\varepsilon>0, N_{\varepsilon}(x)$ contains an element of $E$ distinct from $x$. The derived set of $E$, denoted $E^{*}$, consists of all accumulation points of $E$, and the closure of $E$, denoted $\bar{E}$, is defined by $\bar{E}=E \cup E^{*}$. The set $E$ is closed if $E^{*} \subseteq E$ or, equivalently, if $\bar{E}=E$. The derived set of every set $E$ is closed, i.e., $E^{* *} \subseteq E^{*}$. The set $V \subseteq[0, s]$ is said to be dense in $[0, s]$ if $\bar{V}=[0, s]$.

THEOREM 2.1. Suppose that $V \subseteq[0, s]$ and that $V$ satisfies (2.1), (2.2), and (2.3). Then $V$ is either dense in $[0, s]$, or finite. 
Proof. We show that any infinite subset $V$ of $[0, s]$ satisfying (2.1)-(2.3) is dense in $[0, s]$, or, equivalently, that if $0<a<b<s$, then there exists $v \in V$ such that $a<v<b$. Since $V$ is infinite and $[0, s]$ is compact, $V$ has at least one accumulation point in $[0, s]$. If 0 is among the accumulation points, then there exists $u \in V$ such that $0<u<\min \{a,(b-a) / 2\}$. If we then set $v:=(\lfloor a / u\rfloor+1) u$, it follows that $a<v<b$ and, by (2.3), that $v \in V$. It turns out, however, that it is always the case that $0 \in V^{*}$. For $V^{*}$ is a nonempty set of real numbers bounded below by 0 , and thus admits a greatest lower bound $x \geq 0$. Since $V^{*}$ is closed, $x \in V^{*}$. We now show that if $x>0$, this leads to a contradiction.

Case 1. Suppose that $s=k x$, where $k$ is a positive integer. Let $\left(x+\varepsilon_{i}\right)$ be a sequence of distinct elements of $V$ such that $\varepsilon_{i} \rightarrow 0$ as $i \rightarrow \infty$. (i) If $\left(\varepsilon_{i}\right)$ contains a subsequence $\left(\varepsilon_{i_{j}}\right)$ of negative terms (as will always be the case, for example, when $k=1$ ), then $s-k\left(x+\varepsilon_{i_{j}}\right)=\left|k \varepsilon_{i_{j}}\right| \geq 0$, and so $k\left(x+\varepsilon_{i_{j}}\right) \in V$ by (2.3), and $s-k\left(x+\varepsilon_{i_{j}}\right) \in V$ by (2.2). It follows that 0 is an accumulation point of $V$. (ii) If $\left(\varepsilon_{i}\right)$ does not contain a subsequence of negative terms, then $k \geq 2$, and for $i$

sufficiently large, $\delta_{i}:=s-(k-1)\left(x+\varepsilon_{i}\right)=x-(k-1) \varepsilon_{i} \geq 0$, whence $(k-1)\left(x+\varepsilon_{i}\right) \in V$ by $(2.3)$ and $\delta_{i} \in V$ by (2.2). Then, for $i$ sufficiently large, $s-k \delta_{i}=k(k-1) \varepsilon_{i} \in(0, s)$, whence $k \delta_{i} \in V$ by (2.3) and $s-k \delta_{i} \in V$ by (2.2). But $\delta_{i} \rightarrow x$, and so $s-k \delta_{i} \rightarrow 0$, as $i \rightarrow \infty$. It follows again that 0 is an accumulation point of $V$.

Case 2. Suppose that there exists an integer $k$ such that $k x<s<(k+1) x$. Since $x$ is an accumulation point of $V$, there exists a sequence $\left(x+\varepsilon_{i}\right)$ of distinct points in $V$ such that $\varepsilon_{i} \rightarrow 0$ as $i \rightarrow \infty$. Then for $i$ sufficiently large, it would be true that $k\left(x+\varepsilon_{i}\right)<s$, whence $k\left(x+\varepsilon_{i}\right) \in V$ by (2.3) and $s-k\left(x+\varepsilon_{i}\right) \in V$ by (2.2). But then $s-k\left(x+\varepsilon_{i}\right) \rightarrow s-k x$ as $i \rightarrow \infty$, and so there exists an accumulation point $y$ (namely, $y=s-k x$ ) of $V$ such that $y<x$.

\section{APPENDIX B}

The proof of Theorem 3.2 builds on a result of Bradley and Wagner, which involves a further axiomatic restriction on allocation aggregation.

Zero Unanimity Preservation (Z). For all $j \in\{1, \ldots, m\}$, and all $A \in A(n, m ; V)$, $A_{j}=\mathbf{0} \Rightarrow F(A)_{j}=0$. 
THEOREM BW. Suppose that $V$ is a finite subset of $[0, s]$ that satisfies the closure conditions (2.1)-(2.3). If $m \geq 3$, an AAM $F: A(n, m ; V) \rightarrow A(m ; V)$ satisfies I and $\mathrm{Z}$ if and only if it is dictatorial.

Proof. See Bradley and Wagner [2].

THEOREM 3.2. Suppose that $V$ is a finite subset of $[0, s]$ that satisfies the closure conditions (2.1)-(2.3). If $m \geq 3$, an AAM $F: A(n, m ; V) \rightarrow A(m ; V)$ satisfies I if and only if it is dictatorial or imposed.

Proof. Sufficiency: obvious. Necessity: By I there exist functions $f_{j}: V^{n} \rightarrow V$ such that, for all $A \in(n, m ; s, V), F(A)_{j}=f_{j}\left(A_{j}\right)$ and $\sum_{j=1}^{m} f_{j}\left(A_{j}\right)=s$. For all $X \in V^{n}$ and $j=1, \ldots, m$, let

$$
g_{j}(X):=f_{j}(X)-f_{j}(\mathbf{0})
$$

The functions $g_{j}$ are identically equal to a function that we shall henceforth denote simply by $g$. For suppose that $j_{1}, j_{2} \in[m]:=\{1, \ldots, m\}$ and $j_{1} \neq j_{2}$. Choose any $j_{3} \in[m]-\left\{j_{1}, j_{2}\right\}$ and consider the $s$-allocation matrices $A$, with $A_{j_{1}}=X, A_{j_{2}}=\mathbf{0}, A_{j_{3}}=\mathbf{s}-X$, and $A_{j}=\mathbf{0}$ for any other $j$; and $B$, with $B_{j_{1}}=\mathbf{0}, B_{j_{2}}=X, B_{j_{3}}=\mathbf{s}-X$, and $B_{j}=\mathbf{0}$ for any other $j$. Since $\sum_{j=1}^{m} f_{j}\left(A_{j}\right)=s=\sum_{j=1}^{m} f_{j}\left(B_{j}\right)$, it follows immediately that $f_{j_{1}}(X)-f_{j_{1}}(\mathbf{0})=f_{j_{2}}(X)-f_{j_{2}}(\mathbf{0})$. Note that $g: V^{n} \rightarrow\{0, \pm k s / r: k=1, \ldots, r\}$ for some positive integer $r$. Moreover, if $X, Y, X+Y \in V^{n}$, then

$$
g(X+Y)=g(X)+g(Y) .
$$

For the $s$-allocation matrices $A=(X|Y| \mathbf{s}-X-Y|\mathbf{0}| \cdots \mid \mathbf{0})$ and $B=(X+Y|\mathbf{0}| \mathrm{s}-X-Y|\mathbf{0}| \cdots \mid \mathbf{0})$ yield $f_{1}(X)+f_{2}(Y)=f_{1}(X+Y)+f_{2}(\mathbf{0})$. Subtracting $f_{1}(\mathbf{0})$ from each side of this equation yields $g_{1}(X+Y)=g_{1}(X)+g_{2}(Y)$, and hence (B.2), since $g_{1}=g_{2}=g$. We now consider two possible cases.

Case 1. Suppose that $g(\mathbf{s})=s$. Then $f_{j}(\mathbf{s})-f_{j}(\mathbf{0})=s$ for $j=1, \ldots, m$. But $f_{j}(X) \in V=\{0$, $k s / r: k=1, \ldots, r\}$, and so it must be the case that $f_{j}(\mathbf{s})=s$ and $f_{j}(\mathbf{0})=0$ for $j=1, \ldots, m$. Hence $F$ satisfies $\mathrm{Z}$ as well as $\mathrm{I}$, and is dictatorial by Theorem BW.

Case 2. Suppose that $g(\mathbf{s})<s$. By the extension of (B.2) to $r$ summands, it follows that $g(\mathbf{s})=r g(\mathbf{s} / r)$, and so $g(\mathbf{s} / r)<s / r$, i.e., $g(\mathbf{s} / r) \in\{0,-k s / r: k=1, \ldots, r\}$. If $g(\mathbf{s} / r)=-k s / r$, then $g(\mathbf{s})=-k s$, which is outside the set of possible values of $g$ if $k \geq 2$. On 
the other hand, if $g(\mathbf{s})=-s$, then $f_{j}(\mathbf{s})-f_{j}(\mathbf{0})=-s$ for $j=1, \ldots, m$, and so $f_{j}(\mathbf{s})=0$ and $f_{j}(\mathbf{0})=s$ for $j=1, \ldots, m$. But if $A=(\mathbf{s}|\mathbf{0}| \mathbf{0}|\cdots| \mathbf{0}), F(A)$ is not an $s$-allocation row vector, since $\sum_{j=1}^{m} f_{j}\left(A_{j}\right) \geq 2 s$. So $g(\mathbf{s} / r)=0$.

Now associate with the function $g$ the functions $g^{\langle i\rangle}, i=1, \ldots, n$, defined for all $x \in V$ by $g^{\langle i\rangle}(x)=g(0, \ldots, 0, x, 0, \ldots, 0)$, where $x$ occupies the $i^{\text {th }}$ position in the preceding vector. Clearly, $g\left(x_{1}, \ldots, x_{n}\right)=g^{\langle 1\rangle}\left(x_{1}\right)+\cdots+g^{\langle n\rangle}\left(x_{n}\right)$ for all $\left(x_{1}, \ldots, x_{n}\right) \in V^{n}$. In particular,

$$
g(\mathbf{s} / r)=g^{\langle 1\rangle}(s / r)+\cdots+g^{\langle n\rangle}(s / r)=0 .
$$

For all $x \in V$ and $i=1, \ldots, n, g^{\langle i\rangle}(x) \in\{0, \pm k s / r: k=1, \ldots, r\}$, and by (B.2), if $x, y, x+y \in V$, then

$$
g^{\langle i\rangle}(x+y)=g^{\langle i\rangle}(x)+g^{\langle i\rangle}(y)
$$

By (B.3), if any $g^{\langle i\rangle}(s / r) \neq 0$, then some $g^{\langle i\rangle}(s / r)=k s / r$, where $k \in\{1, \ldots, r\}$. By the extension of (B.4) to $r$ summands, it follows that $g^{\langle i\rangle}(s)=k s$, which is outside the set of possible values of $g^{\langle i\rangle}$ if $k \geq 2$. On the other hand, if $g^{\langle i\rangle}(s)=s$, then for all $j=1, \ldots, m$, $f_{j}(0, \ldots, 0, s, 0, \ldots, 0)-f_{j}(\mathbf{0})=s$, and so $f_{j}(\mathbf{0})=0$. If $A=(\mathbf{s}|\mathbf{0}| \cdots \mid \mathbf{0})$, then $f_{1}(\mathbf{s})+f_{2}(\mathbf{0})+\cdots+f_{m}(\mathbf{0})=s$, whence $f_{1}(\mathbf{s})=s$, and so $g(\mathbf{s})=f_{1}(\mathbf{s})-f_{1}(\mathbf{0})=s$, contrary to our assumption that $g(\mathbf{s})<s$. It follows that $g^{\langle i\rangle}(s / r)=0$ for all $i=1, \ldots, n$, and hence that $g^{\langle i\rangle}(k s / r)=0$ for $k=0,1, \ldots, r$, i.e., that $g^{\langle i\rangle}(x)=0$ for all $x \in V$. So $g(X)=0$ for all $X=\left(x_{1}, \ldots, x_{n}\right) \in V^{n}$, whence $f_{j}(X)=f_{j}(\mathbf{0})$ for all $X \in V^{n}$ and all $j=1, \ldots, m$. So for every $s$ allocation matrix $A, F(A)=\left(f_{1}(\mathbf{0}), \ldots, f_{m}(\mathbf{0})\right)$, and so $F$ is imposed.

\section{Notes}

1. More formally, if $\Omega$ is any countable set, a mass function is a mapping $m: \Omega \rightarrow[0, \infty)$ such that $\sum_{\omega \in \Omega} m(\omega)=s$, for some fixed $s>0$. Each mass function $m$ on $\Omega$ induces a measure $M: 2^{\Omega} \rightarrow[0, \infty)$, with $2^{\Omega}$ denoting the set of all subsets of $\Omega$, and $M(E):=\sum_{\omega \in E} m(\omega)$ for each $E \subseteq \Omega$. When $s=1, m$ is called a probability mass function, usually denoted by $p$, and $M$ is called a probability measure, usually denoted by $P$. In this context, subsets of $\Omega$ are called events. As an alternative to this set-theoretic formulation of probability, which is favored by most mathematicians, philosophers often prefer to conceive of a probability measure as defined on an algebra of propositions, in which case the atoms of that algebra correspond to elements of $\Omega$. 
2. A probability mass function $p$ may, for example, be subject to the requirement that some pair of events $E$ and $F$ be independent with respect to its associated probability measure $P$, that $p$ or $P$ satisfy certain upper or lower bounds on their values, or that the expected value $E(X):=\sum_{\omega \in \Omega} X(\omega) p(\omega)$ of some random variable $X: \Omega \rightarrow \mathrm{R}$ should take some fixed value $\mu$. Allocations of the costs or benefits among the parties of a collaborative enterprise are ideally selected subject to upper and lower bounds designed to discourage the defection of any subset of the parties from the original group. The study of when and how such allocations may be constructed is the subject of cooperative game theory. See, for example, Shapley [14].

3. The term "independence" is derived from Arrow's independence of irrelevant alternatives condition [3], which dictates that the societal ordering of two policy alternatives should depend only on how individual voters order those two alternatives, taking no account of how many of the other alternatives are ranked above, between, or below the two in question. In the context of aggregating probability mass functions or measures, the term "independence" is perhaps not the best choice of terminology. Indeed, it is often replaced by the term "state-wise aggregation," or "proposition-wise aggregation." We shall use all three of these terms interchangeably in this paper, as well as the term "entity-wise aggregation."

4. Alternatively, the various $s$ - allocations might arise from a single individual evaluating the variables on the basis of $n$ different criteria.

5. Note that the quantities $f_{j}\left(A_{j}\right)$ must sum to $s$ without recourse to any normalization.

6. In order for this case to obtain it is necessary and sufficient (given that condition I holds) that $F$ satisfy zero unanimity preservation, i.e., that $f_{j}(\mathbf{0})=0$ for $j=1, \ldots, m$. See Lehrer and Wagner [12, Theorem 6.4] .

7. Given a fixed set $\Omega$ of possible states of the world, an integer $n \geq 2$, and a sigma algebra $S$ of subsets of $\Omega$, and designating by $P(S)$ the set of all probability measures on $S$, McConway calls a mapping $C_{S}: P(S)^{n} \rightarrow P(S)$ a consensus function for $S$. Here, as usual, $C_{S}\left(\pi_{1}, \ldots, \pi_{n}\right)$ denotes the "consensual" probability measure produced by the consensus function $C_{S}$ from the "profile" of probability measures $\left(\pi_{1}, \ldots, \pi_{n}\right)$ assessed by individuals $i=1, \ldots, n$. McConway's innovation is to consider the entire class $\mathbf{C}=\left\{C_{S}: S\right.$ is a sigma algebra on $\left.\Omega\right\}$. This enables him to define what he terms the marginalization property of $\mathbf{C}$, i.e., the property that, for every sigma algebra $S$ on $\Omega$, for each profile $\left(\pi_{1}, \ldots, \pi_{n}\right) \in P(S)^{n}$, for each sub-sigma algebra $T$ of $S$, and for each event $E \in T, \quad C_{S}\left(\pi_{1}, \ldots, \pi_{n}\right)^{(T)}(E)=C_{T}\left(\pi_{1}^{(T)}, \ldots, \pi_{n}^{(T)}\right)(E)$. Here, if $\pi \in P(S), \pi^{(T)}$ designates the marginalization (i.e., the restriction) of $\pi$ to $T$. If the family $\mathbf{C}$ has the marginalization property, then consensus formation commutes with marginalization. McConway 
proves that $\mathbf{C}$ has the marginalization property if and only if, for every sigma algebra $S$ on $\Omega$, for every profile $\left(\pi_{1}, \ldots, \pi_{n}\right) \in P(S)^{n}$, and for every event $E \in S, C_{S}\left(\pi_{1}, \ldots, \pi_{n}\right)(E)$ depends only on $E$ and the numbers $\pi_{1}(E), \ldots, \pi_{n}(E)$. If $C_{S}\left(\pi_{1}, \ldots, \pi_{n}\right)(E)=0$ whenever $\pi_{1}(E)=\cdots=\pi_{n}(E)=0$ (cf. note 6 supra), then the consensual probability of $E$ no longer depends on $E$, but only on the numbers $\pi_{1}(E), \ldots, \pi_{n}(E)$, and is in fact a weighted arithmetic mean of these numbers, precisely as in Lehrer and Wagner [12].

8. More precisely, if $p \in X$, then $\neg p \in X$ unless $p$ is of the form $\neg q$, in which case it is required that $q \in X$. In the latter case, ' $\neg p$ ' refers to $q$ rather than to $\neg \neg p$.

9. If $p$ and $\neg p$ were both atoms of $X$, then (1) $p \neq q$ or (2) $p \vDash \neg q$, and (3) $\neg p$ F $q$ or (4) $\neg p=\neg q$. If (1) and (3), then $q$ is a tautology. If (1) and (4), then $p$ and $q$ are logically equivalent. If (2) and (3), then $p$ and $\neg q$ are logically equivalent. If (2) and (4), then $q$ is a contradiction. Thus in each case $p$ and $q$ would be in trivial dependence.

10. But see Wagner [15], where merely requiring that an aggregation method for probability profiles produce a set function $\lambda$ satisfying $\lambda(E \cup F) \geq \lambda(E)+\lambda(F)-\lambda(E \cap F)$ for all events $E$ and $F$ entails aggregation by weighted arithmetic averaging (so that the output of aggregation is again a probability measure, and nothing is gained).

11. Simply aggregate the $n$ values assigned by individuals to each entity by weighted arithmetic averaging using weights $w_{i}=k_{i} / k$, where the numbers $k_{i}$ are nonnegative integers and $k_{1}+\cdots+k_{n}=k$. It is preferable that $k \geq n$, so that it is possible to assign each individual positive weight.

12. It is one thing to restrict individuals allocating a sum of money to different projects to assigning, say, multiples of $\$ 10,000$ to each project, with the aim of arriving at a consensual allocation in which projects are allocated multiples of $\$ 100$. But asking individuals to artificially limit their probability assignments to a particular valuation domain seems contrary to the goal of arriving at the most informed (or best calibrated) consensual probability assignments. In the case of aggregating zero-one judgments over a set of pairwise contradictory, exhaustive propositions, it is not even clear how one would interpret the weighted averages of such judgments that would be allowed by enlarging the codomain $\{0,1\}$. Formally, such averages would of course constitute a probability mass function on the set of propositions in question. But they would lack foundation, either as objective or as subjective probabilities.

13. As argued in [17] and [18], it is difficult to imagine how one can devise an aggregation method that applies to every logically possible profile without proceeding entity-by-entity (resp., proposition-by-proposition or state-by-state).

\section{References}


1. Aczél, J., Ng, C.T. \& Wagner, C. (1984). Aggregation theorems for allocation problems. SIAM Journal on Algebraic and Discrete Methods, 5, 1-8.

2. Aczél, J. \& Wagner, C. (1980). A characterization of weighted arithmetic means. SIAM Journal of Algebraic and Discrete Methods, 1, 259-260.

3. Arrow, K. (1950). A difficulty in the concept of social welfare. The Journal of Political Economy, 58, 328-346.

4. Balinski, M. \& Young, H. (1985). The apportionment of representation. Proceedings of Symposia in Applied Mathematics 33 (Fair Allocation), American Mathematical Society, 1-29.

5. Black, D. (1968). The Theory of Committees and Elections, Cambridge University Press, Cambridge, England.

6. Bradley, R. \& Wagner, C. (2012). Realistic opinion aggregation: Lehrer-Wagner with a finite set of opinion values. Episteme, 9, 91-99.

7. Brown, D. (1975). Aggregation of preferences. Quarterly Journal of Economics, 89, 456-469.

8. Dietrich, F. (2006). Judgment aggregation: (im)possibility theorems. Journal of Economic Theory, 126, 286-298.

9. Dietrich, F. \& List, C. (2010). The aggregation of propositional attitudes: Towards a general theory. Oxford Studies in Epistemology, volume 3, Oxford University Press, Oxford.

10. Genest, C. (1984). Pooling operators with the marginalization property. Canadian Journal of Statistics, 12, 153-163.

11. Genest, C. \& Zidek, J. (1986). Combining probability distributions: a critique and annotated bibliography. Statistical Science, 1, 114-148.

12. Lehrer, K. \& Wagner, C. (1981). Rational Consensus in Science and Society, D. Reidel Publishing Co., Dordrecht.

13. McConway, K.J. (1981). Marginalization and linear opinion pools. Journal of the American Statistical Association, 76, 410-414.

14. Shapley, L. (1971). Cores of convex games. International Journal of Game Theory, 1, 11-26.

15. Wagner, C. (1989). Consensus for belief functions and related uncertainty measures. Theory and Decision, 26, 295-304.

16. (2010). Jeffrey conditioning and external Bayesianity. Logic Journal of the $I G P L, 18,336-345$, DOI:10.1093/jigpal/jzp063. 
17. (2011). Peer disagreement and independence preservation. Erkenntnis, 74, 277 288.

18. (2012). Universality and its discontents. Balkan Journal of Philosophy, 4, 39-44.

\section{Acknowledgment}

The authors wish to thank Franz Dietrich for suggesting that Theorem BW in Appendix B might be true whenever the valuation domain $V$ fails to be dense in $[0, s]$. We are also grateful to an anonymous referee for suggesting how to apply Theorem 3.2 to the case of infinite atomic agendas. 